\title{
Sensory Input Drives Multiple Intracellular Information Streams in Somatosensory Cortex
}

\author{
Andrea Alenda, ${ }^{1}$ Manuel Molano-Mazón, ${ }^{1}$ Stefano Panzeri, ${ }^{2}$ and Miguel Maravall ${ }^{1}$ \\ ${ }^{1}$ Instituto de Neurociencias de Alicante, Universidad Miguel Hernández-Consejo Superior de Investigaciones Científicas, 03550 Sant Joan d'Alacant, Spain, \\ and 'Italian Institute of Technology, Robotics, Brain, and Cognitive Sciences Department, 16163 Genova, Italy
}

Stable perception arises from the interaction between sensory inputs and internal activity fluctuations in cortex. Here we analyzed how different types of activity contribute to cortical sensory processing at the cellular scale. We performed whole-cell recordings in the barrel cortex of anesthetized rats while applying ongoing whisker stimulation and measured the information conveyed about the time-varying stimulus by different types of input (membrane potential) and output (spiking) signals. We found that substantial, comparable amounts of incoming information are carried by two types of membrane potential signal: slow, large (up-down state) fluctuations, and faster $(>20 \mathrm{~Hz})$, smaller-amplitude synaptic activity. Both types of activity fluctuation are therefore significantly driven by the stimulus on an ongoing basis. Each stream conveys essentially independent information. Output (spiking) information is contained in spike timing not just relative to the stimulus but also relative to membrane potential fluctuations. Information transfer is favored in up states relative to down states. Thus, slow, ongoing activity fluctuations and finer-scale synaptic activity generate multiple channels for incoming and outgoing information within barrel cortex neurons during ongoing stimulation.

\section{Introduction}

Stable perception arises from cortical sensory responses that are profoundly variable across trials. Much of this variability can be explained by patterns of activity fluctuations shared by a large fraction of neurons in a region (Zohary et al., 1994; Amzica and Steriade, 1995; Arieli et al., 1995, 1996; Buracas et al., 1998; Azouz and Gray, 1999; Lampl et al., 1999; Tsodyks et al., 1999; Anderson et al., 2000; Petersen et al., 2003; Deweese and Zador, 2004). Rather than being just noise, such fluctuations may have important functions, e.g., modulating stimulus processing (Buzsaki, 2006; Destexhe and Contreras, 2006; Destexhe et al., 2007). One pattern of fluctuations prominent during slow-wave sleep and under anesthesia involves changes between a less-active "down" state, characterized by hyperpolarized membrane potentials, and an active "up" state, in which neurons are depolarized (Metherate

Received Dec. 14, 2009; revised June 23, 2010; accepted June 24, 2010.

Financial support was provided by Spanish Ministry of Science and Innovation (Grant BFU2008-03017/BFI, c0funded by the European Regional Development Fund); CONSOLIDER Grant (SD2007-00023), Human Frontier Science Program Grant RG0043/2004, the Italian Institute of Technology (Brain Machine Interface project of the Department of Robotics, Brain, and (ognitive Sciences), Compagnia di San Paulo, and European Commission Coordination Action ENINET Contract Number LSHM-CT-2005-19063. We thank A. Belitski for help with filtering, C. Magri for help with implementation of the information theoretic analysis, M. DeWeese, M. Häusser, M. London, and L. Martínez for help with in vivo patch recordings, M. López de Armentia for experimental advice, R. Petersen and G. Foffani for useful discussions, and M. Diamond, L. Martínez, L. Menéndez de la Prida, and R. Petersen for comments on this manuscript.

M.M. designed the project. A.A. performed the experiments. S.P. developed the information analysis. M.M.-M., S.P., and M.M. analyzed and interpreted the data. M.M. drafted the manuscript, and all authors contributed to it.

Correspondence should be addressed to Miguel Maravall, Instituto de Neurociencias de Alicante, Universidad Miguel Hernández-Consejo Superior de Investigaciones Científicas, Campus de San Juan, Apartado 18, 03550 Sant Joan d'Alacant, Spain. E-mail:mmaravall@umh.es.

A. Alenda's present address: Institute of Behavioural Neuroscience, University College London, 26 Bedford Way, London WC1H OAP, UK.

DOI:10.1523/JNEUROSCI.6174-09.2010

Copyright $\odot 2010$ the authors $\quad 0270-6474 / 10 / 3010872-13 \$ 15.00 / 0$ and Ashe, 1993; Steriade et al., 1993; Cowan and Wilson, 1994; Amzica and Steriade, 1995; Lampl et al., 1999; Anderson et al., 2000; Buzsaki, 2006; Destexhe et al., 2007). Barrel cortex sensory responses are strongly affected by activity state (Erchova et al., 2002; Petersen et al., 2003; Castro-Alamancos, 2004; Sachdev et al., 2004; Haslinger et al., 2006; Hasenstaub et al., 2007; Reig and Sanchez-Vives, 2007); conversely, sufficiently strong whisker stimuli can themselves trigger transitions between down and up states (Hasenstaub et al., 2007; Reig and Sanchez-Vives, 2007). Moreover, spontaneous and sensory-evoked activity fluctuation patterns can have similar spatiotemporal structure (Tsodyks et al., 1999; Kenet et al., 2003; Petersen et al., 2003; MacLean et al., 2005; Luczak et al., 2009).

These findings suggest that, in cortex, interactions between stimulus-dependent responses and emergent activity play a fundamental role in sensory processing (Buonomano and Maass, 2009; Ringach, 2009), yet how different types of activity interact at the cellular scale remains poorly understood. Here we aimed to explore these cellular-level interactions by measuring the membrane potential of barrel cortex neurons during ongoing, extended stimulation. We wanted to quantify sensory encoding by both subthreshold and spiking signals.

In principle, methods based on information theory (Shannon, 1948) (for review, see Borst and Theunissen, 1999; Quian Quiroga and Panzeri, 2009) can quantify how sensory responses interact with variations in activity. Measuring the stimulus information carried by membrane potential and spiking signals can give insight into the impact of different types of activity on sensory processing. However, application of information methods to cortical in vivo membrane potential data has been complicated by statistical estimation issues: information estimates become biased when data are undersampled, requiring the use of appropri- 
ate bias reduction techniques (Panzeri et al., 2007). Here we addressed this by extending recent advances in information estimation for graded signals (Montemurro et al., 2007) to wholecell barrel cortex recordings in vivo. We found that slow fluctuations and finer-scale synaptic activity provide multiple intracellular streams for information flow in the barrel cortex.

\section{Materials and Methods}

\section{Experiments}

All procedures complied with Society for Neuroscience, European, Spanish, and institutional policies for the care and use of animals in research. Wistar rats $(n=23$; male and female) were anesthetized with urethane $(1.5 \mathrm{~g} / \mathrm{kg}$, i.p.) and placed in a stereotaxic device (Narishige) while their body temperature was maintained at $37^{\circ} \mathrm{C}$ using a homeothermic heating pad (FHC). Eyelid and hindpaw reflexes were monitored throughout the experiments, and refresher urethane doses were added if necessary. Animals were aged postnatal day 30 (P30) to P40 (mean of P35) and weighed between 72 and $140 \mathrm{~g}$ (mean of $105 \mathrm{~g}$ ).

After performing a small ( $\sim 2 \mathrm{~mm}$ on the side) craniotomy centered at anteroposterior $2.5 \mathrm{~mm}$ and lateral $5.2 \mathrm{~mm}$ relative to bregma, draining the cisterna magna, and reflecting the dura, we obtained patch-clamp recordings in whole-cell mode according to standard blind procedures (Margrie et al., 2002; DeWeese, 2007). After breaking through to wholecell mode, the recording was switched to current-clamp configuration. Pipettes had 3-7 M $\Omega$ resistance and were filled with solution containing the following (in $\mathrm{mM}$ ): $130 \mathrm{~K}$-methylsulfonate, $10 \mathrm{Na}$-phosphocreatine, 10 HEPES, $4 \mathrm{MgCl}_{2}, 4 \mathrm{Na}_{2}$-ATP, $3 \mathrm{Na}$-ascorbate, and $0.4 \mathrm{Na}_{2}$-GTP. No attempt was made to correct for junction potentials. Recordings were digitized at $8 \mathrm{kHz}$ [Axon Multiclamp 700B; CED 1401, Signal 3 software (Cambridge Electronic Design)]. Recording depth was estimated using the reading of the micromanipulator (Sutter Instruments MP-225). Depths were in the range of 345-1700 $\mu \mathrm{m}$ (mean $1150 \mu \mathrm{m}$ ): most recordings were estimated as being within layer $\mathrm{V}$.

Several vibrissae (E1-E4, D1-D4, C1-C4, gamma, delta; cut to 10 $\mathrm{mm}$ ) were jointly introduced into a grid made of rigid plastic tubes glued to a piezoelectric bender (Physik Instrumente); the tip of the bender was located $\sim 3 \mathrm{~mm}$ from the vibrissa pad. Joint stimulation with a common waveform simplified interpretation of the results, because it implied that information was always about a unique time series, as opposed to the case in which different time series are simultaneously applied to different vibrissae; stimulating an array of whiskers instead of a single whisker maximized data collection, because the stimulus was ready to begin immediately after patching a cell.

The stimulator had a dynamic range of $400 \mu \mathrm{m}$ and was stimulated with a purpose-built amplifier (Physik Instrumente). Motion direction was manually adjusted for each neuron to produce the clearest onset response.

Stimuli were $15 \mathrm{~s}$ duration stochastic waveforms created in Matlab (MathWorks) as Gaussian-distributed white noise sampled at $10 \mathrm{kHz}$. They were then low-pass filtered (pass band, $\sim 0-200 \mathrm{~Hz}$ ) by convolving with a Gaussian kernel (1.6 ms width) (see Fig. 1A). This restricted frequencies to well under the nonlinear resonance range of the stimulator. The overall amplitude of the waveform was modulated over a factor of 2 using a slowly changing envelope (cycle duration, $5 \mathrm{~s}$ ). Whisker exploration of surfaces is often irregular and extended and can generate complex sequences of vibrations (Arabzadeh et al., 2005; Lottem and Azouz, 2008, 2009; Ritt et al., 2008; Wolfe et al., 2008; Jadhav et al., 2009); our final stimulus contained a wide range of frequencies similar to those of texture-modulated whisker deflections, as well as slower changes corresponding to modifications in overall amplitude or context. Maximum speed during ongoing stimulation was $130 \mathrm{~mm} / \mathrm{s}$; speed SD was $16.4 \mathrm{~mm} / \mathrm{s}$.

\section{Data analysis}

All analysis was performed in Matlab (MathWorks). Of the full set of successful recordings $(n=26)$, a subset $(n=8)$ was selected for full analysis based on fulfillment of the following conditions: (1) recording parameters (spike height and shape, input resistance) were stable throughout successive recording periods for a total recording time be- tween 8 and $45 \mathrm{~min}$; (2) up and down states were well enough separated, compared with the width of $V_{m}$ histogram peaks, to allow clear classification (see below); and (3) there was no evidence of biases in the information calculation arising from undersampling (i.e., an inadequate number of trials; see below). Spikes were extracted by thresholding the voltage waveform. Average firing rates varied widely across neurons (0.32-20 spikes/s, median of 3.9 spikes/s).

Classification into up and down states. Neurons usually displayed prominent up and down states (see Fig. $1 B$ ). Up and down states were identified by dual thresholding on the membrane potential histogram of a recording and further applying a duration threshold (Hasenstaub et al., 2007). First, we searched for a trough between the peaks in the membrane potential histogram; the trough usually fell close to the mean voltage of the recording (supplemental Fig. S1, available at www.jneurosci.org as supplemental material). Next, we searched for thresholds on either side of the trough by identifying where the slope fell off to a factor of 0.1 times its maximum; this location was within the distance from the trough to the inflection point of the histogram rising toward the peak. Finally, we classified a period as being in the up state if the membrane potential crossed the upper threshold from below and then spent at least $100 \mathrm{~ms}$ above the upper threshold without dropping under the lower threshold (i.e., brief drops under the upper threshold were permissible as long as the lower threshold was not breached). A similar criterion (but with 50 ms duration threshold) was applied to the down state. Periods not fulfilling the conditions for an up or down state were left unclassified. Figure $3 A$ (top trace) shows an example trace with classified up and down states.

Transitions to up or down states were counted and binned at $100 \mathrm{~ms}$ resolution. The number of transitions observed in the first $100 \mathrm{~ms}$ bin after stimulus onset was compared with the distribution of transitions during spontaneous activity. The significance of the deviation from baseline was determined either using a normal fit to baseline or (if the spontaneous distribution was non-normal by the Lilliefors test) an empirical cumulative distribution function. Consistent with previous accounts (Anderson et al., 2000; Hasenstaub et al., 2007; Reig and Sanchez-Vives, 2007), the rate of transitions from periods classified as down states to periods classified as up states was significantly increased by stimulus onset ( $p<10^{-7}$ for all neurons but one).

Membrane potential response filtering and processing. Our analyses required estimates of the information content of subthreshold membrane potentials and of spiking responses to be based on a common set of experimental trials. To allow this, before estimating membrane potential information, each trace was median filtered to remove spikes $(7.1 \mathrm{~ms}$ window). Because median filtering does not completely eliminate contributions of spike-associated conductances, this could potentially lead to artifacts when estimating information in the membrane potential, a problem that would worsen with increasing levels of firing. However, we found no effect of firing rate on either overall membrane potential information (Pearson's $r^{2}=0.029 ; p=0.69$ ) or fine-scale membrane potential information (see Results for explanation of this term; Pearson's $r^{2}=$ $0.061, p=0.56)$.

Information estimates for the membrane potential were based on this median-filtered version of the signal; likewise, additional manipulations of the membrane potential were performed on the median-filtered version. We note that, because this is a processed version of the signal, our information estimates might not capture the full information jointly conveyed by knowledge of all aspects and sources of membrane potential fluctuations (see below for additional details on information). Our strategy was to select and compare different signals as candidate information carriers rather than to attempt to extract all possible information.

To understand whether the information encoded by the medianfiltered membrane potential is dominated by low-frequency fluctuations, we obtained low-pass filtered versions of the signal by using a Kaiser filter (Kayser et al., 2009) (sharp transition bandwidth, $1 \mathrm{~Hz}$; small ripple, $0.01 \mathrm{~dB}$; high stop-band attenuation, $60 \mathrm{~dB}$ ) and progressively decreasing the cutoff frequency from 40 to 20 to $5 \mathrm{~Hz}$ (for examples, see Fig. $3 A$ ). We used these data to compare whether information decreased when decreasing the cutoff frequency (see Results). To explore whether smaller membrane potential fluctuations at higher frequencies also conveyed information, we computed a fine-scale high-frequency signal by 
subtracting the low-pass filtered traces $(20 \mathrm{~Hz}$ cutoff) from the original median-filtered trace, thus preserving structure in the range beyond 20 $\mathrm{Hz}$. We chose this approach over high-pass filtering because traces resulting from high-pass Kaiser filtering had ringing artifacts that made interpretation difficult. Phase and amplitude traces were determined by first taking the Hilbert transform of the filtered trace and then computing the phase and amplitude of the transformed signal.

Because we were interested in characterizing responses to ongoing stimulation, we concentrated on response epochs after rapid adaptation was over (Maravall et al., 2007). To do so, we avoided transient onset responses by discarding the first $5 \mathrm{~s}$ of each response, leaving a $10 \mathrm{~s}$ response period per trial.

Information estimation. The mutual (Shannon) information (Shannon, 1948) between a set of stimuli $S$ and a set of responses $R$ is defined as

$$
I(S ; R)=-\sum_{\mathbf{r}} P(\mathbf{r}) \log _{2} P(\mathbf{r})+\sum_{\mathbf{s}} P(\mathbf{s}) \sum_{\mathbf{r}} P(\mathbf{r} \mid \mathbf{s}) \log _{2} P(\mathbf{r} \mid \mathbf{s}),
$$

where $P(\mathbf{s})$ is the probability of presenting stimulus $\mathbf{s}, P(\mathbf{r} \mid \mathbf{s})$ is the probability of observing response $\mathbf{r}$ to stimulus $\mathbf{s}$, and $P(\mathbf{r})$ is the probability of observing response $\mathbf{r}$ across all stimuli. With base 2 logarithms, $I(S ; R)$ has units of bits: when observation of a response reduces uncertainty by a factor of 2 on average, mutual information is equal to 1 bit. When calculating information about the stimulus segment presented (de Ruyter van Steveninck et al., 1997; Strong et al., 1998), this equation simplifies to

$$
I=\left\langle\sum_{\mathbf{r}} P(\mathbf{r} \mid t) \log _{2} \frac{P(\mathbf{r} \mid t)}{P(\mathbf{r})}\right\rangle_{t}
$$

where $P(\mathbf{r} \mid t)$ is the probability of observing response $\mathbf{r}$ at time $t$, time is divided into segments of length $T$, and the brackets denote averaging over time segments. For a stimulus-sensitive neuron, the time-conditional distribution $P(\mathbf{r} \mid t)$ varies with time (see Fig. $2 A$ ). Conversely, for a stimulus-insensitive neuron, $P(\mathbf{r} \mid t)$ does not vary with time and is equal to $P(\mathbf{r})$ for all $t$ : in this case, $I=0$, consistent with the idea that uncertainty about the stimulus is not reduced by observing the response of the neuron. Unlike other measures of correlation between random variables, information has the advantage of capturing all nonlinear dependencies of any statistical order that may be present in the data.

An additional important aspect of information-based approaches is that they provide insight into single-trial processing, in that they measure the average decrease in uncertainty afforded by a response on a single trial. This reproduces an essential aspect of the task faced by an animal trying to discriminate environmental input. Measuring information in the laboratory does require multiple repetitions of a given stimulus or condition, and achieving a reasonable number of repetitions is the main limiting factor when designing experiments.

We used a method based on direct sampling of the time-dependent response probability distributions (see Fig. 2A) (de Ruyter van Steveninck et al., 1997; Strong et al., 1998). This direct method specifically measures information about which dynamic stimulus time segment elicited the response. As discussed previously (de Ruyter van Steveninck et al., 1997; Strong et al., 1998), the method has the advantage that it does not make any assumption as to which specific dynamic features of the stimulus triggered the neural response and so can potentially capture the information about all possible dynamical stimulus features presented experimentally. However, because the method involves a choice of time resolution $T$ when dividing up the stimulus into segments (see below), in practice it may not capture information about dynamic features that cannot be defined or computed within the observation time window $T$. For this reason, information measures computed with this method are to be considered as relative to the specified time window $T$; below, we always report the value of $T$ used to compute information.

With this caveat, direct sampling produces correct estimates of information about stimulus time, contrary to other methods that make simplifying assumptions as to response probability distributions (e.g., a Gaussian shape) and thus provide upper or lower bounds on information (Borst and Theunissen, 1999). However, precisely because this method involves estimation of response histograms, it is particularly data intensive and susceptible to undersampling bias. Its successful use therefore requires bias reduction methods, usually consisting of extrapolation (Strong et al., 1998; Juusola and de Polavieja, 2003; Panzeri et al., 2007) coupled to additional steps (Panzeri et al., 2007). Moreover, the direct method does not prescribe how best to extract (decode) the information present on a given individual trial, nor does it specify how much information was present on the trial.

In practice, we estimated information by taking the following steps. In the first step, we selected the response quantity of interest: medianfiltered or low-pass filtered membrane potential; amplitude and phase of each filtered membrane potential; spike count; spike timing relative to stimulus; spike timing relative to the membrane potential slow fluctuation phase.

In the second step, we computed the value of the relevant response quantity for each segment of length $T$ (ranging between 5 and $20 \mathrm{~ms}$ ) and each trial (see Fig. $2 A$ ). For graded variables (membrane potential signals), this value was obtained by taking the mean value (circular mean for phase values) over the duration of the segment ( $T$ ) (see Fig. $2 B$ ) and then discretizing into $N_{R}$ approximately equipopulated bins (except for phase values, which were discretized into equally spaced bins). Note that temporal binning effectively imposed a low-pass filter during the signal, because it reduced fluctuations to a single value over each segment: the resulting information estimates are likely to be especially conservative for higher-frequency signals. For each segment, we then estimated the distribution of possible response values $P(\mathbf{r} \mid t)$ by binning over repeated trials; $P(\mathbf{r})$ was estimated by binning over all trials and segments. The number of bins $N_{R}$ is a free parameter of the analysis and must be chosen on the basis of empirical considerations. On the one hand, lower values of $N_{R}$ give lower resolution, potentially missing out on some of the information carried by the neural signal (see Figs. $2 A, 4 A$ ), but decrease the sampling problems inherent to information calculation. On the other hand, higher values of $N_{R}$ increase resolution, thus potentially capturing more information, but they also complicate sampling and lead to greater upward bias in information estimates, caused by limited sampling (Panzeri et al., 2007). Here we determined the value of $N_{R}$ by varying it parametrically and then choosing the lowest value of $N_{R}$ that provided convergent information in neural responses, with higher values of $N_{R}$ providing no significant genuine increase in information (see Fig. $2 C$ ) (described in detail below). For neurons in our dataset, information in membrane potential signals converged for $N_{R}=10$ (see Fig. 2C) (supplemental Fig. S2, available at www.jneurosci.org as supplemental material). Furthermore, although the results presented were all obtained using $N_{R}=10$, we verified that all qualitative results remained unaltered when using $N_{R}=8$ and 12 (data not shown).

For spike count, the relevant value was the number of spikes in each segment. Because the response collected in individual segments was considered separately, this quantification ignored the information potentially present in the correlations between spikes emitted in different segments. In practice, because we used short $T$ values (20 ms or less), there was seldom more than one spike in each segment (time bin). We tested whether using a binary spike count quantification ( 0 or 1 , meaning spikes or no spikes in any given segment; $N_{R}=2$ ) caused information loss, and the result was that any loss was negligible (for $T=20 \mathrm{~ms}, p=$ 0.84 , paired $t$ test). We therefore used binary counts throughout. For spike timing relative to the stimulus, each stimulus segment of length $T$ was divided into a series of $L$ smaller windows of size $\Delta t=T / L$ (Strong et al., 1998). In this potentially richer code, each response, instead of consisting of a 0 or 1 , was a binary "word" of length $L$ : for example, for $T=$ $20 \mathrm{~ms}$ and $\Delta t=5 \mathrm{~ms}(L=4)$, there were $N_{R}=2^{4}$ possible responses. Finally, for spike timing relative to slow fluctuation phase, we labeled each spike with the phase of the $20 \mathrm{~Hz}$ low-pass filtered membrane potential at the time of firing; phases were circularly averaged across the segment when the spike was emitted (as for membrane potential data, see above) and then binned into $N_{R}=4$ uniformly spaced values. This simplified binning could only underestimate the information gain from phase labeling, leading to conservative conclusions. Contrary to the membrane potential signals, which were resolved at $N_{R}=10$ (see Fig. $2 C$ ), labeling with a more finely resolved slow fluctuation phase did not 
lead to increased information ( $p=0.90$, Friedman's test). Thus, with labeling, the number of response categories increased from $N_{R}=2$ (binary spike count) to $N_{R}=5$ (inactive and four possible active categories corresponding to different phases) (Montemurro et al., 2008; Kayser et al., 2009).

In the third step, we corrected for biases caused by limited data sampling (Panzeri et al., 2007). Bias errors can be particularly serious when estimating the stimulus-conditional response probabilities $P(\mathbf{r} \mid t)$ : estimation of unconditional response probabilities $P(\mathbf{r})$ is based on a larger effective sample, equal to the number of repeats (trials) times the number of stimulus segments in each repeat. Biases are approximately inversely proportional to the effective sample size and proportional to the number of possible responses $N_{R}$. Response quantities with larger numbers of possible values included membrane potentials (see Fig. 2C), phaselabeled spikes, and, especially, joint fine-scale and slow fluctuation signals as well as spike timing relative to the stimulus. For calculations involving these quantities, we implemented a recent procedure (Montemurro et al., 2007) based on a "shuffling" information estimator that greatly reduces the bias of the information carried by multidimensional responses (Panzeri et al., 2007). We finally applied an asymptotic bias correction procedure to extrapolate to infinite sample size and subtract out bias: we used both the "Panzeri-Treves" (Panzeri and Treves, 1996) and "quadratic extrapolation" (Strong et al., 1998) procedures and found that they gave essentially identical results.

Prediction of information bias from increased resolution. As discussed above, apparent increases in information from more finely resolved responses can in fact result from an increased bias. To set the appropriate resolution for our membrane potential dataset, our logic was to start from a given number of bins $N_{R}$, increase the number, and check whether the resulting variation in information could be accounted for by a change in bias. If so, nothing was gained from using the finer resolution. If, on the contrary, there was a gain in information beyond the predicted bias increase, this suggested that the improved resolution was relevant.

The artificial increase of information as a result of worsening of bias can be predicted as follows. According to considerations by Panzeri et al. (2007), for a certain $N_{R}$, bias is given by

$$
\text { Bias }=\frac{(S-1)\left(N_{R}-1\right)}{2 N_{\text {tot }} \ln 2},
$$

where $S$ is the number of stimulus segments, and $N_{\text {tot }}$ is the total number of samples (which is equal to the number of stimulus segments times the number of trials per segment, $\left.N_{\text {tot }}=S \times N\right)$. Assuming that $S$ is large, as in this study ( $S$ ranged between 500 and 2000), the equation becomes

$$
\text { Bias }=\frac{\left(N_{R}-1\right)}{2 N \ln 2} .
$$

Thus, bias grows linearly with the number of bins, i.e., with binning resolution. If the number of bins increases from $N_{R}$ to $N_{R}{ }^{\prime}$, the increase in bias will lead to a predicted difference in information equal to

$$
I_{\text {diff }}(\text { Bias })=\frac{\left(N_{R}^{\prime}-N_{R}\right)}{2 N \ln 2} .
$$

For the experiments presented here, $N$ (the number of trials or repeats) ranged between 45 and 170 (median of 74 ), giving a predicted $I_{\text {diff }}$ (Bias) of $\sim 0.04$ bits (median). In practice, bias correction techniques eliminate a major part of this difference. Still, as shown in Figure $2 C$, changes in information in the median-filtered membrane potential when going from $N_{R}=10$ to higher values showed a strongly saturating dependence rather than a linear one, with $N_{R}=10$ bins capturing at least $95 \%$ of the asymptotic information. Furthermore, the measured information difference from $N_{R}=10$ to $N_{R}^{\prime}=14$ was approximately an order of magnitude smaller (median of 14\%) than could be accounted for by uncorrected increases in bias. We concluded that information measurements for the median-filtered membrane potential had converged by $N_{R}=10$. Results were equivalent for the information in low-pass filtered and fine-scale signals (supplemental Fig. S2, available at www.jneurosci.org as supplemental material).
Effects of noise and stimulus artifacts. Because stimuli were generated as an electrical signal fed to a piezoelectric bender, there was a chance that stimulus signals could contaminate the membrane potential recording. Contamination from a prolonged, graded stimulus waveform with a broad frequency spectrum can be harder to discriminate and filter out than contamination from a discrete, sharp waveform. We reasoned that such an artifact would be likely to be almost identical across repeated trials, because it would amount to a scaled version of the stimulus; therefore, it could cause an overestimate of information. We therefore attempted to quantify the size of possible artifacts.

To do so, we first computed, for each recording, the cross-correlogram between the stimulus and the average response. (Because an artifact would be present in all trials, we expected that averaging responses would reduce trial-to-trial noise and keep only the artifact plus the consistent elements of the response.) We found that the experimental crosscorrelogram was never centered at $0 \mathrm{~ms}$; instead, it was shifted by a few $(\sim 1-2)$ milliseconds in the causal direction (i.e., with the response retarded with respect to the stimulus). This shift in the cross-correlogram ruled out contamination by an unfiltered version of the stimulus; however, it was still possible that contamination occurred after the stimulus was effectively filtered by circuitry.

To predict the effects of contamination by the filtered stimulus artifact, we next applied several filter designs to a simulated white-noise experimental stimulus. After filtering the stimulus, we added random noise, creating a set of simulated "responses" consisting of the filtered stimulus plus noise. We then treated these responses identically to the experimental ones, taking their average and computing a cross-correlogram. We found that the slight shift in the peak of the experimental crosscorrelogram could be reproduced by these simulations; however, we were never able to reproduce the overall shape of the cross-correlogram. More interestingly, we used the simulations to explore the dependence of the cross-correlogram and of mutual information on the level of noise added to the simulated filtered stimulus artifact. The result was that, although the peak amplitude of the cross-correlogram was relatively robust to added noise, the mutual information between the stimulus and the noisy filtered responses dropped off very sharply as noise increased. Mutual information became negligible when the noisy responses were still a recognizable (i.e., significantly correlated, although noisy) version of the original stimulus. Conversely, in our experimental data, the crosscorrelogram peaks between stimulus and response were always smaller than the range explored in our simulations, yet the mutual information was always non-negligible (i.e., was significantly higher than that for shuffled responses). This indicates that the information carried in our experimental responses was too large to be accounted for by contamination from a filtered stimulus waveform. Thus, although we cannot rule out that stimulus artifacts might sometimes have been present in our recordings, we can rule out that they significantly contributed to information.

Information conveyed during up and down states. Because up and down states are not time-locked to the stimulus, i.e., not triggered at fixed times on every trial, we could not use time labeling to compute the information contained within the up and down periods. Instead, we adopted an approach based on shuffling responses within up or down periods, as follows. To estimate the membrane potential information contained within, e.g., up states, we temporally shuffled all membrane potential segments contained within the up states of each trial. This eliminated the information carried while in up states, because all possible stimulusrelated structure in the membrane potential during up states was eliminated. Hence, the information in this shuffled signal was reduced by an amount equal to the information contained within the up periods. By subtracting the remaining information from the original value, we therefore obtained an estimate of the information contained within up-state periods. Next, we repeated this computation, this time shuffling a number of segments identical to that within the up states but chosen at random, and took the ratio of the information lost by shuffling the up-state periods to the information lost by random shuffling. This normalization step allowed us to account for the relative amount of time spent in up states, because these took up a variable fraction of the duration of each recording (range of 10-39\%). We followed an identical approach for down-state information. 
Statistical testing and presentation. When pooling and comparing quantities across the population, we checked for normality using the Lilliefors test; depending on its result, we then used either parametric or nonparametric tests as detailed in Results. Tests were twotailed, except those for possible gains in information from registering spikes with improved time resolution, because spike count information is a lower bound on spike timing information. (All comparisons in which one-tailed tests were used also gave significant results under two-tailed testing.) In the text, all population data are presented as median and range; in the figures, data are displayed either for all neurons or as box plots showing medians, quartiles, and outliers.

\section{Results}

Slow membrane potential fluctuations convey stimulus information

Using patch recordings in urethaneanesthetized rats, we quantified subthreshold and suprathreshold responses of neurons $(n=8)$ in the barrel cortex, while stimulating whiskers with complex, prolonged fluctuating waveforms (Gaussian-distributed, filtered noise, see Materials and Methods; 15 s duration) (Fig. 1A). This stimulus design allowed us to present a wide-ranging, unbiased sample of whisker fluctuations and to analyze responses to ongoing stimulation. Whisker position fluctuated on a fast timescale $(\sim 5 \mathrm{~ms}$ correlation time). Stimuli were repeated over $45-170$ trials.

During ongoing stimulation, membrane potentials displayed large excursions between a depolarized (up) state and a hyperpolarized (down) state (Fig. 1B) (see also Fig. 3A). At first glance, this pattern of slow fluctuations between up and down states appeared to be spontaneously generated, with no obvious temporal locking to stimuli (Fig. $1 B$, colored lines represent different trials). However, closer examination of traces suggested that certain stimulus events evoked repeatable responses (Fig. $1 B$, arrows and asterisks; corresponding stimulus plotted directly above in $A$ ). On a subset of these events (Fig. $1 B$, arrows), every trial showed evidence of strong synaptic activation, and this consistent activation of synaptic inputs caused an enhanced probability of transitioning to an up state. Thus, the membrane potential, and in particular its slow fluctuations, was modulated by whisker stimulation (Fig. $1 B, C$ ).

If the responses of a neural system are influenced by a sensory stimulus, there will be some degree of systematic correlation between stimulus and response. If this is true, then reading out the responses will provide information about the stimulus. Based on this idea, one can use information measurements to determine which aspects of neuronal activity are related to the stimulus and could, potentially, serve the function of encoding sensory signals (Borst and Theunissen, 1999; Quian Quiroga and Panzeri, 2009). As mentioned above, in our experiments, neuronal membrane potentials were modulated by whisker stimulation. We quantified this modulation
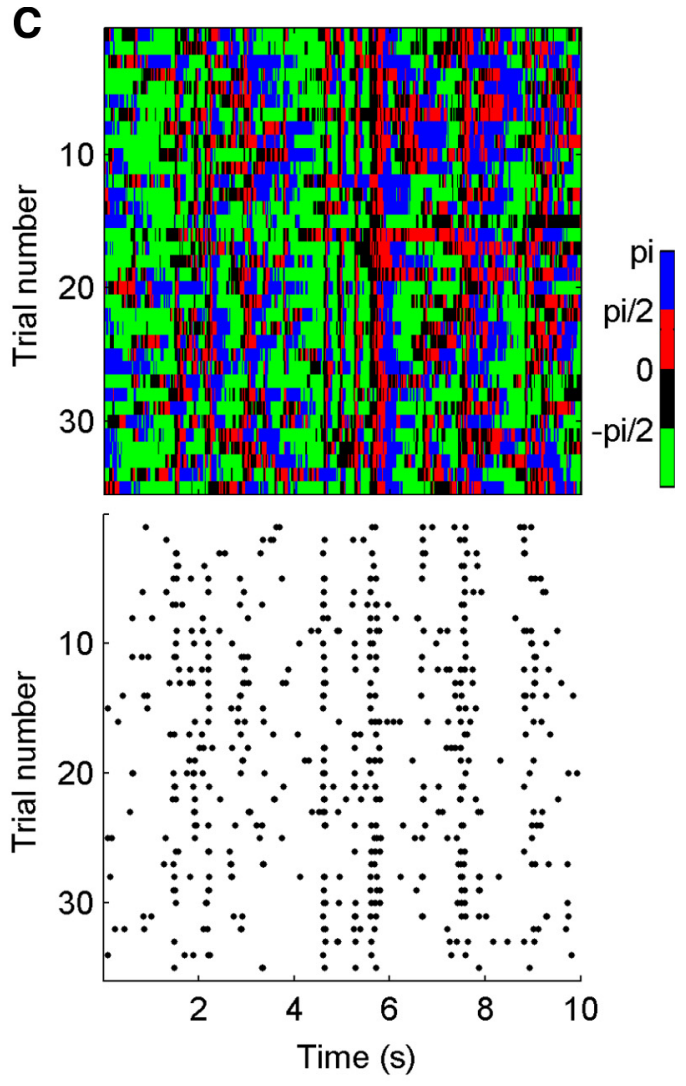

\section{0}

Figure 1. Whole-cell responses to ongoing whisker stimuli. $\boldsymbol{A}$, Whisker stimulus waveform (arbitrary units; timescale at bot列 stimulus elicited a $V_{m}$ response on every trial, often leading to a down- up transition and sometimes to a spike. C, Stimulus modulation of membrane potential phase and of spiking. Top, Time course of membrane potential phase over $10 \mathrm{~s}$ of continuing

by measuring the mutual information between the membrane potential and the stimulus.

Formally, the mutual information between two stochastic variables (Shannon, 1948) (referred to as "information" in the following) is a measure of the reduction in uncertainty about one of the variables (e.g., the sensory input) that an observer experiences upon sampling the other variable (e.g., the output of a neuron). To estimate the information that responses contained about the time-varying stimulus, we divided up the stimulus into nonoverlapping segments of length $T$, each of which was taken to be a different "stimulus value" (Fig. 2A) (de Ruyter van Steveninck et al., 1997; Strong et al., 1998). This method for computing information quantifies the reduction in uncertainty about the identity of the stimulus segment (i.e., about which stimulus segment was being presented) that one would gain by measuring various aspects of the neuronal response (Fig. 2A) (further discussed in Materials and Methods).

We first measured the information conveyed by the membrane potential signal after median filtering to eliminate spikes (Fig. 3A) (see Materials and Methods; for simplicity, we denote this signal as $\left.\mathrm{mf} V_{m}\right)$. Our computations involved taking the mean of the signal over a segment window duration $T=20 \mathrm{~ms}$ and then 
A
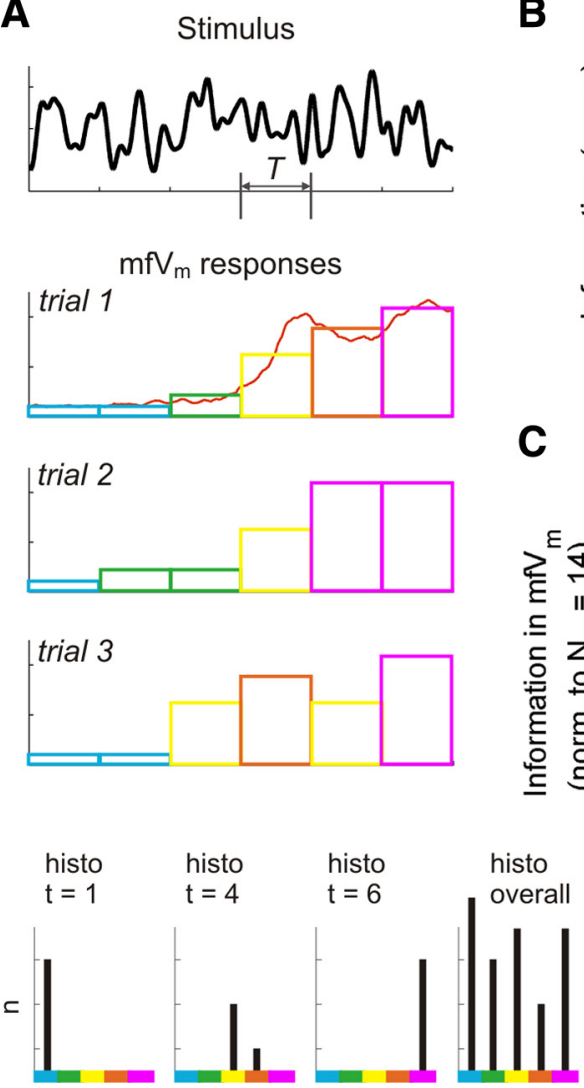

Figure 2. Information estimation using the "direct" method. $\boldsymbol{A}$, Schematic of the direct method for estimating information in time-varying responses. A stimulus sequence of $120 \mathrm{~ms}$ duration (top) is repeated and responses measured across several trials (middle). The median-filtered membrane potential $\left(\mathrm{mfV}_{\mathrm{m}}\right)$ response for trial 1 (red curve) is averaged over segments of length $T=$ $20 \mathrm{~ms}$; the average of each segment is binned into one of several categories (represented by rectangles in different colors). In this example, the procedure was repeated across three trials: the resulting histograms (bottom) show the number of occurrences of each binned value (different colors) at specific time points $(t=1,4,6)$ or overall. The responses were informative about the stimulus, because different time points in the stimulus evoked very different response distributions. In other words, although responses were widely distributed overall, the set of responses at any given time was tightly constrained, and this made the responses predictive of the stimulus segment. $\boldsymbol{B}$, Dependence of binned $\mathrm{mfV}_{m}$ information on temporal bin size. Bin size, $T$, is the length of the time window for defining stimulus segments and averaging $\mathrm{mfV}_{m}$. Colored lines, Individual neurons; thick black line, Average. For segment lengths $T=5-20 \mathrm{~ms}, \mathrm{mfV}_{m}$ information values were approximately at a plateau. $T=20 \mathrm{~ms}$ gave $103 \%$ as much information (median) as $T=5 \mathrm{~ms}$ (range of $99-112 \%$ ). These segment lengths were comparable with membrane integration time constants. Averaging over longer timescales did not appreciably degrade information as long as $T$ remained below the period of slow fluctuations ( $\sim 100 \mathrm{~ms}$ ). C, Dependence of $\mathrm{mfV}_{m}$ information on response binning resolution. Colored lines, Example neurons accepted into final dataset; thin gray line, example neuron discarded from final dataset; thick black line, average for accepted neurons; diagonal dashed line, linear dependence. Information should converge with increasing resolution $N_{R}$, saturating at some finite resolution: this is the behavior shown by the well sampled neurons (in colors). For these neurons, our results suggest that $\mathrm{mfV}_{m}$ information did not substantially benefit from a binning resolution beyond $N_{R}$ of $\sim 10$. Lack of convergence (quasi-linear growth) for the example discarded neuron was likely caused by a small number of trials (20), which led to limited-sample bias: bias is proportional to $N_{R^{\prime}}$ producing spuriously increasing information estimates. Thus, this and other neurons with limited trials were discarded. For the accepted experiments, qualitative results were not affected by the choice of $N_{R}$ : results for $N_{R}=8$ and 12 followed the same trends as for $N_{R}=10$.

binning the resulting response value into one of $N_{R}=10$ different categories; however, all qualitative results were robust to the specific choice of these parameters (Fig. $2 B, C$ ) (details in Materials and Methods). Different neurons varied widely in the amount of stimulus information conveyed by their binned $\mathrm{mfV}_{m}$ (Fig. 3B) (median of 0.15 bits; range of $0.063-0.41$ bits for $T=20 \mathrm{~ms}$ ): therefore, they were driven by this particular stimulus to varying degrees. Interestingly, these differences in information were uncorrelated with differences in firing rate (see Materials and Methods, Membrane potential response filtering and processing) and were also uncorrelated with recording depth (Pearson's $r^{2}=0.033$; $p=0.67)$ or cortical layer ( $p=0.39$, Kruskal-Wallis).
The phase component of $\mathrm{mf} V_{m}$ - the relative position within a fluctuation $\mathrm{cy}$ cle, including, e.g., upswings and downswings-appeared to be significantly driven by the stimulus (Fig. 1C). We thus wondered whether $\mathrm{mf} V_{m}$ phase might be particularly rich in information. We computed the phase and amplitude components of the $\mathrm{mfV}_{m}$ signal by taking its Hilbert transform (example phase values are shown in Figs. $1 C, 3 A$ ). Just as for $\mathrm{mf} V_{m}$, we computed the information in the resulting quantities by averaging each signal over a segment window duration $T=20 \mathrm{~ms}$ and then binning the resulting value into one of $N_{R}=10$ categories; again as for $\mathrm{mf} V_{m}$, qualitative results were robust to parameter choice. The result was that binned phase was significantly more informative than binned amplitude (Fig. 3C) (phase: median of 0.19 bits; range of 0.069-0.58 bits; amplitude: median of 0.069 bits; range of $0.035-0.26$ bits; $p=$ 0.0040 , paired $t$ test). Phase conveyed more information than amplitude whether the $\mathrm{mf} V_{m}$ of a neuron conveyed large or small quantities of information.

Visual inspection of response traces suggested that the $\mathrm{mf} V_{m}$ signal was dominated by activity contained in a relatively low-frequency range (under $\sim 20 \mathrm{~Hz}$ ). Indeed, low-pass filtering at $20 \mathrm{~Hz}$ captured most of the structure in $\mathrm{mf} V_{m}$, including the overall shape of transitions between down and up states (Fig. 3A) [this is consistent with the fact that the highestpower contributions to $\mathrm{mf} V_{m}$ were from its low-frequency components (supplemental Fig. S3, available at www. jneurosci.org as supplemental material)]. However, the range of fluctuations with the highest power need not be the one carrying the most information (Belitski et al., 2008). For example, adding a large amount of high-frequency noise to a lowfrequency information-bearing signal will result in a signal with a more informative low-frequency component, despite the greater power at high frequencies. Thus, to determine whether the high-power, slow fluctuation frequencies in the $\mathrm{mf} V_{m}$ signal were important for transmitting information, we low-pass filtered the signal with different cutoff frequencies $(5,20$, or 40 $\mathrm{Hz}$ ) (Fig. $3 A$ ) and computed the resulting information using the same approach as for the original signal. The result was that lowpass filtering did not reduce the information content of the binned signals (Fig. 3D) ( $p=0.99$, ANOVA). We further decomposed each low-passed signal into phase and amplitude by taking its Hilbert transform, as described above for the full $\mathrm{mf} V_{m}$. Just as for the median-filtered signal, the phase component of each binned low-passed signal conveyed significantly more information than the corresponding amplitude component (data not shown; $p<0.001$, two-way ANOVA; no interaction between 
A
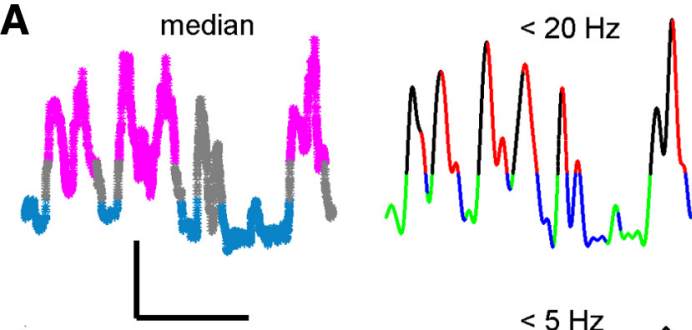

B

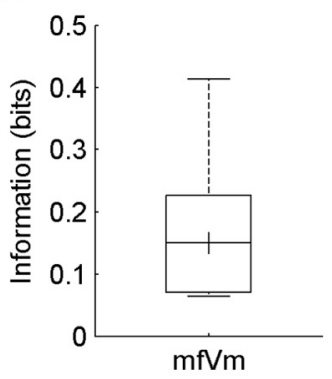

c

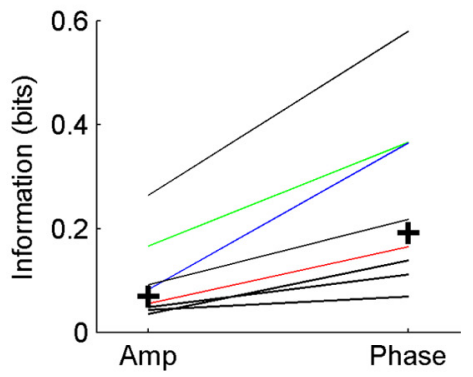

D

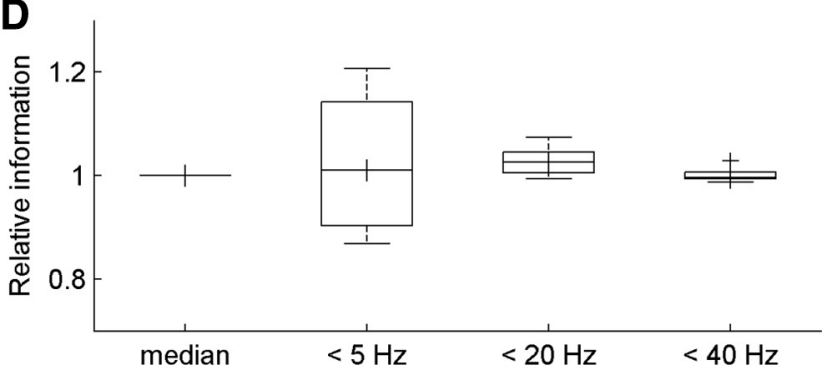

Figure 3. Stimulus information revealed in the filtered membrane potential. $\boldsymbol{A}$, Effect of low-pass filtering on example membrane potential responses (different neuron from examples in Fig. 1). Calibration: $5 \mathrm{mV}, 500 \mathrm{~ms}$. Left, Median-filtered trace: colors denote periods identified as up states (magenta), down states (blue), or neither (gray; including transitions). Right, Same trace after low-pass filtering ( 5 and $20 \mathrm{~Hz}$ cutoffs): colors denote phase, convention as in Figure $1 C . \boldsymbol{B}$, Information conveyed by the median-filtered membrane potential (averaged and binned over a time window $T=20 \mathrm{~ms}$; number of response bins $N_{R}=10$ ). . Information conveyed by the amplitude and phase of the membrane potential's Hilbert transform (parameters as for $\boldsymbol{B}$ ). Crosses, Median values; red, example neuron in Figure $1 B$; green, example neuron in Figure $1 C$; blue, example neuron in $\boldsymbol{A}$; black, other neurons. $\boldsymbol{D}$, Information conveyed by median-filtered and low-pass-filtered membrane potential (respectively, median, 5, 20, and $40 \mathrm{~Hz}$ cutoffs) (parameters as for $\boldsymbol{B}$ ). Information for each neuron was normalized to that in the corresponding median-filtered $V_{m}$ signal (statistical tests described in Results were conducted on the original, non-normalized signals).

factors, $p=0.99)$. In summary, the phase of low-frequency $m f V_{m}$ fluctuations, including up-down-state transitions, was highly informative about ongoing stimulation.

\section{Fine-scale fluctuations convey substantial} independent information

The analysis described above demonstrated that lowfrequency fluctuations in the median-filtered membrane potential carried essentially as much information as the (binned) $\mathrm{mf} V_{m}$ itself, yet a higher-frequency, finer-scale component of synaptic activity was superimposed on the slow fluctuations (for an example, see Fig. $4 A$ ). The above result, namely that low-pass filtering did not reduce information, appeared to
A

B
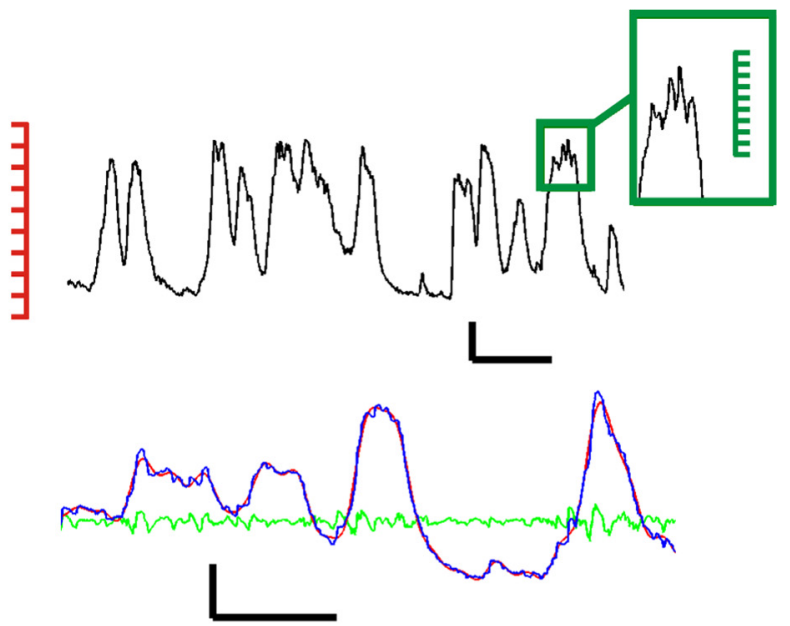

C
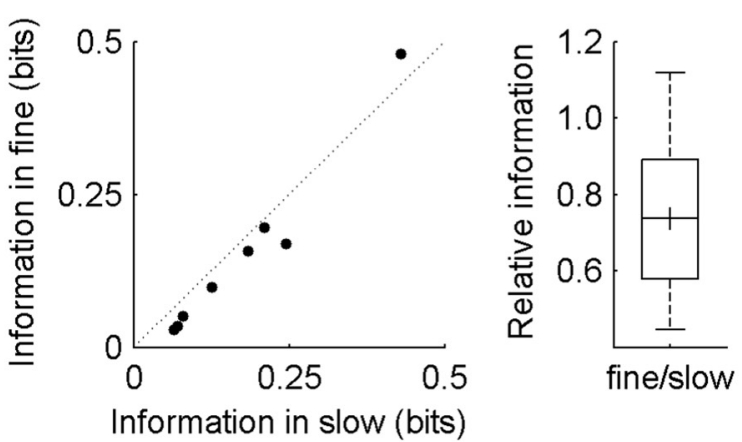

D
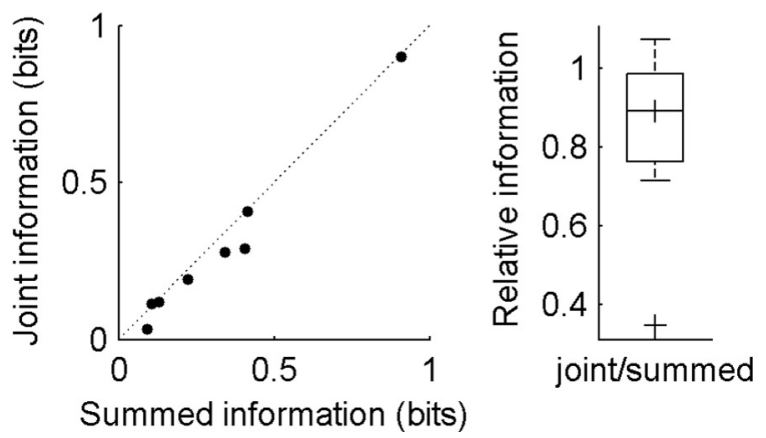

Figure 4. Substantial, independent information contained in fine-scale synaptic activity. $\boldsymbol{A}$, Representation of response resolution for calculations of information (same neuron as Fig. $1 B$ ). A fixed histogram scale appropriate to the magnitude of the whole $\mathrm{mfV}_{m}$ response including large-scale fluctuations (red scale) has insufficient resolution to capture smaller-scale fluctuations (magnified trace within the blown-up region in green). Neurons, however, can readjust their sensitivity, effectively rescaling their input resolution (green scale). Calibration: $5 \mathrm{mV}, 500$ ms. $\boldsymbol{B}$, Example original (median-filtered) data (blue trace), low-pass filtered data ( $20 \mathrm{~Hz}$; red trace), and subtracted fine-scale data (green trace). Calibration: $5 \mathrm{mV}, 200 \mathrm{~ms}$. C, Information in the subtracted, fine-scale signal versus information in the $20 \mathrm{~Hz}$ low-pass-filtered signal $(T=$ $\left.20 \mathrm{~ms} ; N_{R}=10\right)$. Left, Dots, neurons; dotted line, equality. Right, Relative information, normalized for each neuron. $\boldsymbol{D}$, Information in the joint fine-scale and low-pass-filtered $(20 \mathrm{~Hz})$ signals versus sum of the information carried by both signals. Left and right as in C; parameters as for $\boldsymbol{C}$. For points close to the diagonal, joint information was close to the summed information, indicating that the fine-scale and low-pass-filtered signals were close to independent.

imply that, when present in the $\mathrm{mf} V_{m}$ trace together with the lower-frequency components, the finer-scale activity did not increase information. However, we wondered whether the fine-scale structure might in fact convey additional information that was obscured in our analysis by the presence of the larger, slower fluctuations.

This could happen in the following way. Any direct estimate of information relies on binning responses into a histogram (for a 
representation, see Fig. $2 \mathrm{~A}$ ). The resolution of the histogram is limited by two factors: first, the histogram range needs to cover the full range of responses; second, each histogram bin must be large enough to be adequately sampled, because poor sampling can lead to severe biases (see Materials and Methods) (Fig. 2C). In our case, as described previously, the magnitude of the full $\mathrm{mf} V_{m}$ signal was dominated by its low-frequency component, i.e., by the slow fluctuations (Figs. 3A, 4A). Thus, information estimates for the full $\mathrm{mf} V_{m}$ and for low-passed signals involved histograms with a common optimal resolution (bin size). However, this bin size was unlikely to be fine enough to capture the smaller, higherfrequency fluctuations riding on top of the slow component (Fig. $4 A$ ). Hence, sensory information potentially present in the higher-frequency fine-scale fluctuations could be missed by the information analysis, unless the fine-scale activity was considered separately, using histograms with higher resolution (Fig. 4A, green scale at right).

Although information estimates might miss sensory signals in the fine-scale activity because of coarse-scale response binning, this is not a liability shared by neurons. Indeed, neurons integrating their synaptic inputs can readjust their sensitivity, and hence their resolution, via a multitude of adaptive mechanisms: these can render neurons preferentially sensitive to, for example, signals in particular frequency ranges (Azouz and Gray, 1999, 2003; Azouz, 2005; Léger et al., 2005; Prescott and De Koninck, 2005; Higgs et al., 2006; Díaz-Quesada and Maravall, 2008) (Fig. 4A, green scale at right). Thus, neurons can potentially read out information in finer-scale synaptic activity. We therefore decided to extend our information analysis to the finer-scale, higherfrequency component of $\mathrm{mf} V_{m}$. To do this, we filtered out the slow component by taking each median-filtered trace and subtracting from it the corresponding $20 \mathrm{~Hz}$ low-pass filtered version, so that only the higher-frequency fine-scale signal remained. An example (Fig. $4 B$ ) shows how subtracting the 20 $\mathrm{Hz}$ low-pass-filtered signal discarded slow fluctuations in $\mathrm{mf} V_{m}$ yet conserved finer-scale, higher-frequency structure. We then conducted the information analysis adjusting the histogram scale to the resulting fine-scale signal (while maintaining the number of bins, $N_{R}=10$, and the temporal averaging resolution, $T=20 \mathrm{~ms}$ ).

How large was the stimulus information carried by the finescale membrane potential signal compared with that conveyed by the large-scale, low-frequency components? There was a median of $73 \%$ as much information in the binned fine-scale signal as in the binned $20 \mathrm{~Hz}$ low-pass filtered signal (Fig. 4C) (range of 45-112\%). Thus, substantial information was always carried in the fine-scale signal, comparable with that present in the slow fluctuations. We note that the estimate of information in the fine-scale signal was conservative, because of the low-pass filtering effectively imposed by temporal averaging: the true ratio of fine-scale information to slow fluctuation information is likely to be even higher.

We wondered whether the slow and fine-scale signals constituted independent channels for information, i.e., whether they conveyed information independently. To determine this, we computed the joint information in both signals (following the same binning approach as above) and compared this with the summed information. If two channels convey information independently, their joint information is equal to the sum of the information that each conveys separately. If they are redundant, their joint information is less than the summed information; if they are synergistic, their joint information is greater than the sum. For the majority of neurons, the joint information was close, although not equal, to the sum of the slow and fine-scale information (Fig. $4 D$ ): the median ratio of joint to summed information was $89 \%$ (Fig. 4D) (range of $35-107 \%$; 35\% was an outlier, the next smallest value being $72 \%$ ). This indicates that, in the majority of neurons, slow $(<20 \mathrm{~Hz})$ fluctuations and finescale synaptic activity $(>20 \mathrm{~Hz})$ convey essentially independent stimulus information.

\section{Spike information increases when considering timing relative to the stimulus and to membrane potential fluctuations}

We next investigated information in the spiking outputs of the neurons. We first focused on information carried by spike counts, i.e., by changes in spike rate across stimulus segments. As with membrane potential signals, the information that spike counts conveyed about stimulus segment varied widely across neurons (Fig. 5A): using a segment time window $T=20 \mathrm{~ms}$, median information was 0.026 bits with a range spanning $0.012-$ 0.079 bits. For the majority of neurons, increasing the temporal window duration from $T=5 \mathrm{~ms}$ to $T=20 \mathrm{~ms}$ produced an approximately linear increase in spike count information (Fig. $5 A$ ), and a mutual information rate could be defined by computing linear fits to the linear slope. The median information rate computed in this way was $1.4 \mathrm{bits} / \mathrm{s}$ (range of $0.51-3.4 \mathrm{bits} / \mathrm{s} ; n=$ 6). Across neurons, spike count information correlated weakly with average spike rate (Pearson's $r^{2}=0.34 ; p=0.13$ ), indicating that variability in the overall amount of information arose not just from the number of emitted spikes but also from the amount of information carried per spike. Just as for the information in filtered membrane potentials, spike count information did not correlate significantly with recording depth (Pearson's $r^{2}=$ $0.026, p=0.70$ ) or layer ( $p=0.77$, Kruskal-Wallis).

Spike timing can play an important role in encoding whisker stimuli (for review, see Petersen et al., 2009). We thus wondered whether information increased when considering spike timing. By subdividing each stimulus segment of length $T$ into a series of $L$ smaller windows of size $\Delta t=T / L$ (Strong et al., 1998), thus registering the occurrence of spikes with greater precision, we determined whether the temporal structure of spiking responses (i.e., spike timing locked to the stimulus) carried information beyond that in spike counts. Resolving spikes with precision $\Delta t=$ $5 \mathrm{~ms}$, we found that timing information was significantly greater than count information (Fig. $5 B-D$ ) (for $T=10 \mathrm{~ms}$, median gain was 31\%; range of $19-120 \%$; $p<0.001$, one-tailed paired $t$ test; for $T=20 \mathrm{~ms}$, median gain was $72 \%$; range of $40-270 \%$; $p$ $<0.001$, one-tailed paired $t$ test).

Both in the cortex and elsewhere in the nervous system, the phase of the extracellular field potential at the time that a spike is fired can convey additional information to that in spike counts (Huxter et al., 2003, 2008; Montemurro et al., 2008; Kayser et al., 2009). This implies a general principle for information multiplexing: that spike timing locked to activity fluctuations, and not just to the stimulus, is also capable of encoding information (Ahissar and Vaadia, 1990; O'Keefe and Recce, 1993; Hopfield, 1995; Friedrich et al., 2004; Lisman, 2005; Fries et al., 2007; Koepsell et al., 2009, 2010; Nadasdy, 2009; Panzeri et al., 2010). To evaluate whether, similarly, additional information can be gained by considering the membrane potential of a cortical neuron each time it fires a spike, we labeled each spike with the membrane potential phase at the time of firing, computed from the $20 \mathrm{~Hz}$ low-pass filtered signal (binned into $N_{R}=4$ uniformly spaced values, i.e., quadrants). The resulting information was larger than the spike count information (Fig. $5 E, F$ ) (median gain of $13 \%$; range of $10-150 \%$; $p=0.0039$, one-tailed Wilcoxon's 
A
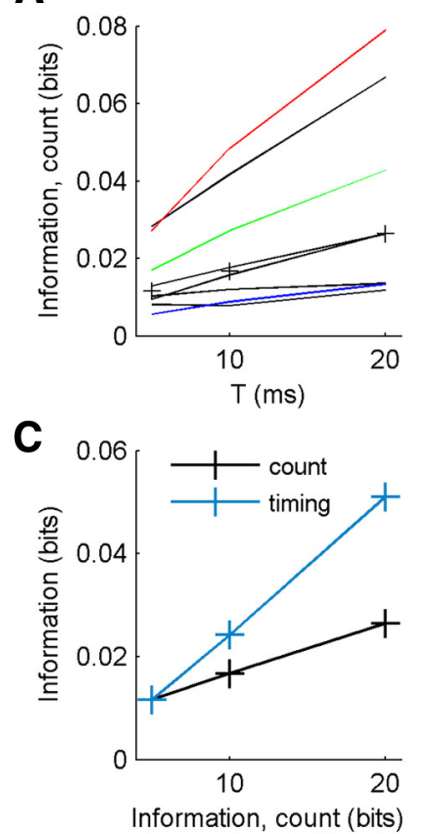

E

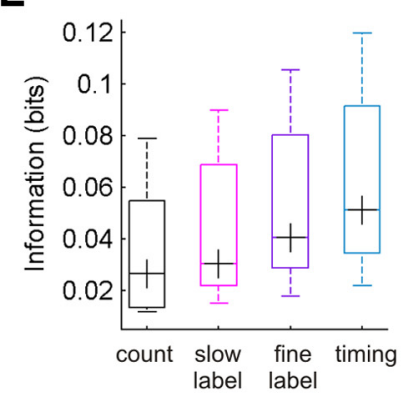

B

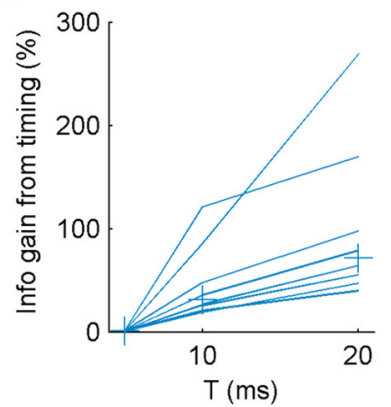

D

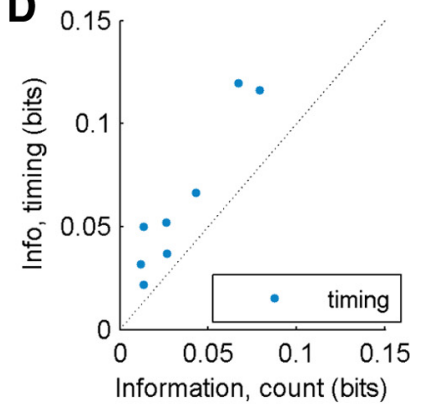

$\mathbf{F}$

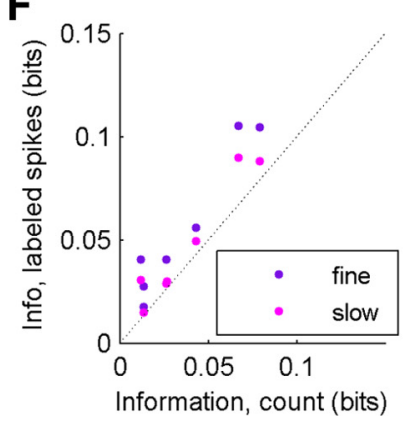

Figure 5. Increased information in spike timing relative to the stimulus and to membrane potential fluctuations. $\boldsymbol{A}$, Information carried by output spike count as a function of $T$. Crosses, Median values; red, neuron in Figure $1 B$; green, neuron in Figure $1 C$; blue, neuron in Figure $3 A$; black, other neurons. Note quasi-linear increase with $T$ in most neurons. $\boldsymbol{B}$, Information gain from spike timing relative to the stimulus $(\Delta t=5 \mathrm{~ms})$. Lines, Neurons; crosses, median values. C, Median information in spike count (black) and spike timing relative to the stimulus (blue) as a function of $T . \boldsymbol{D}$, Information in spike timing relative to the stimulus versus information in spike count. $T=20 \mathrm{~ms}, \Delta t=5 \mathrm{~ms}$. Dots, Neurons; dotted line, equality. $\boldsymbol{E}$, Information in spike count and timing relative both to the membrane potential and to the stimulus. $T=20 \mathrm{~ms}$. Black Count; magenta, timing labeled with $20 \mathrm{~Hz}$ slow oscillation phase; purple, timing labeled with fine-scale membrane potential; blue, spike timing relative to stimulus ( $\Delta t=5 \mathrm{~ms}$ ). Note the increased information from timing in all cases. $\boldsymbol{F}$, Information in spike timing relative to membrane potential signals versus information in spike count. $T=20 \mathrm{~ms}$. Dots, Neurons; dotted line, equality; magenta, spike times labeled with $20 \mathrm{~Hz}$ slow oscillation phase; purple, spike times labeled with fine-scale membrane potential.

signed ranks test), implying that the timing of spikes relative to the phase of slow fluctuations carried information. We next repeated this calculation labeling spikes with the fine-scale membrane potential signal at the time of firing. This again produced a significant increase in information (Fig. $5 E, F$ ) (median gain of $44 \%$; range of $13-240 \%$; $p=0.0022$, one-tailed paired $t$ test).

Information is preferentially transferred in up states

As discussed above, the high stimulus information contained in slow fluctuations corresponding to changes between up and down states implies that these fluctuations can be modulated by an ongoing, prolonged stimulus. We next asked the converse

question: how does the state of activity (up vs down) influence the information conveyed by the membrane and spiking signals: the input and output of the neurons?

To address this, we focused on responses evoked within up or down states. We first examined the fine-scale filtered membrane potential signals described above, which correspond closely to the synaptic activity observed on top of the peaks and troughs of the slow fluctuations (Fig. $4 A, B$ ). To determine the information conveyed specifically within up and down periods, we estimated the information lost by randomly temporally shuffling the filtered membrane potential segments contained within those periods; to account for the relative time spent in up or down states, we compared the information lost during up or down states with that lost by shuffling an identical number of randomly chosen response segments (for additional details, see Materials and Methods).

The result (Fig. 6A) was that the information content of finescale fluctuations (as defined above) during up states was widely variable across neurons but tended to be less than the information during generic (i.e., randomly chosen) periods of the same duration (median of $74 \%$ of generic information; range of 22 $118 \% ; p=0.021$, paired $t$ test). Information content of fine-scale signals during down states was always poorer than during randomly chosen periods (median of $65 \%$; range of $6-81 \%$; $p=$ 0.0021 , paired $t$ test). To interpret these data, note that the randomly chosen periods could include undefined and transition times and therefore could include the synaptic fluctuations responsible for transitioning between up and down states, which are likely to be highly informative.

Information conveyed by spike counts in up or down states was computed using similar logic: we temporally shuffled the spike counts corresponding to up or down states and then normalized as above (Fig. 6B). The information carried by spikes evoked during up states was variable: it was greater than, but comparable with, the information during randomly chosen periods (median of $156 \%$ of generic; range of $43-380 \% ; p=0.079$, paired $t$ test). Spikes in down states carried little information (median of $1.0 \%$; range of $0-32 \%$; $p=0.0078$, Wilcoxon's signed ranks test). The relative information conveyed by the spiking output of the neurons during down states was sharply cut compared with that in their finescale membrane potential fluctuations: often, the ratio of spike count information in down states relative to up states was typically $>10$ times smaller (Fig. $6 C$ ). Thus, the neuronal conversion of membrane potential into spikes shuts down information transfer in down states, mainly through a drastic reduction in the number of spikes emitted (Fig. 6D) (two neurons did not fire at all in down states). Interestingly, for many neurons, the ratio of spike count information in down states relative to up states was above the corresponding ratio of spike rates (Fig. 6D), implying that spikes emitted within down periods were richer than those within up periods.

These results imply that, during prolonged whisker stimulation, while a neuron is in the down state, it communicates (transfers) information extremely selectively, in most cases, only if the stimulus evokes a transition to the up state. Conversely, during up states, neurons transmit spiking information in an ongoing manner. Spiking information is transferred mainly during up states and transitions.

\section{Discussion}

We measured the stimulus information (reduction in uncertainty about a whisker sensory stimulus) conveyed by signals 
A

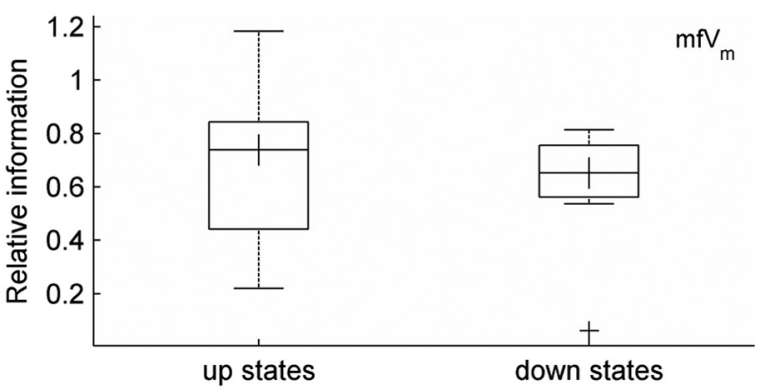

B
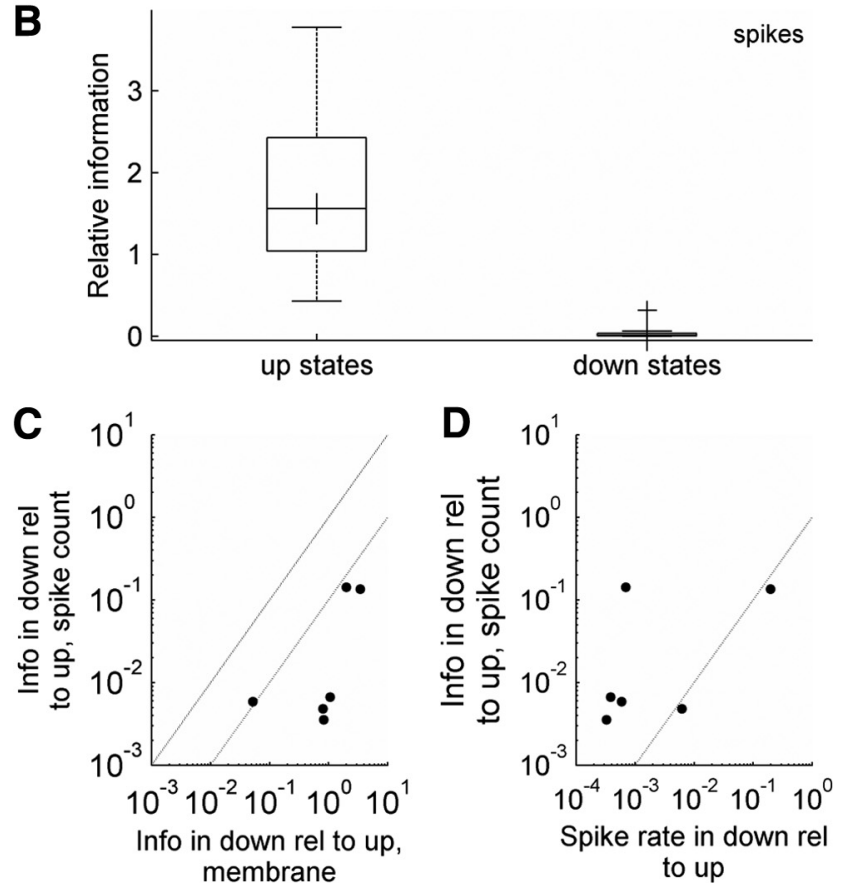

Figure 6. Differences in information transfer as a function of activity state. $\boldsymbol{A}$, Information contained in fine-scale membrane potential fluctuations in up or down states, relative to randomly chosen periods $\left(T=20 \mathrm{~ms} ; N_{R}=10\right)$. $\boldsymbol{B}$, Information conveyed by spike count in up or down states, relative to randomly chosen periods. $\boldsymbol{C}$, Relative spike count information carried in down states and up states (down-state information divided by up-state information), plotted against relative fine-scale membrane potential information normalized in the same way (parameters as for $\boldsymbol{A})$. Dotted lines represent equality $(y=x)$ and $y=x / 10$. Plot includes only six points because two neurons did not fire at all in down-state periods: for those two neurons, the relative information in down states was 0 (note $\log -\log$ coordinates). $\boldsymbol{D}$, Relative spike count information carried in down states and up states as in $\boldsymbol{C}$, plotted against ratio of spike rates in down states and up states. Dotted line, Equality.

extracted from filtered membrane potential of a cortical neuron and by its spike train output. We found that slow, large-scale membrane potential fluctuations and faster, finer-scale fluctuations act as effectively independent, parallel channels for incoming information, that both channels convey substantial information, and that labeling spikes with respect to either channel significantly increases the information in the output of the neuron. Slow large-scale fluctuations represent changes in network activity between up and down states. Because they are continually modulated by an ongoing stimulus, slow changes in activity constitute, in themselves, a code capable of carrying substantial information. However, changes in activity also modulate the information conveyed by other signals.

\section{Different channels for input information}

We found substantial information carried in the phase of slow membrane potential fluctuations. Previous work has established that slow fluctuations are shared across the local network and correlate with local field potential (LFP) phase (Zohary et al., 1994; Amzica et al., 1995; Arieli et al., 1995, 1996; Buracas et al., 1998; Azouz and Gray, 1999; Lampl et al., 1999; Tsodyks et al., 1999; Anderson et al., 2000; Petersen et al., 2003; Deweese and Zador, 2004; Hasenstaub et al., 2007; Saleem et al., 2010). Together with those findings, our data imply that each neuron has access to the phase information contained in LFP activity (Montemurro et al., 2008).

Our results agree with the idea that "spontaneous" collective activity can be modulated by sensory stimuli (Anderson et al., 2000; Kenet et al., 2003; Fiser et al., 2004; Loewenstein et al., 2005; MacLean et al., 2005; Jones et al., 2007; Gao et al., 2009; Luczak et al., 2009). In the barrel cortex, the onset of sufficiently strong stimuli can evoke transitions between down and up states (Hasenstaub et al., 2007; Reig and Sanchez-Vives, 2007). Moreover, pulsed thalamocortical stimulation can trigger sequences of up-down fluctuations similar to spontaneously occurring sequences (MacLean et al., 2005). Our data suggest that an ongoing stimulus continues to modulate membrane potential fluctuations throughout its duration. This implies that sequences of updown transitions observed during prolonged stimulation do not consist entirely of preexisting patterns set off, or triggered, by stimulus onset. Rather, although specific activity sequences can be intrinsically sustained, continuing sensory input appears to modulate or entrain the sequences. This suggests that changes in correlations between different neurons (Poulet and Petersen, 2008) can be driven by sensory input, as well as by internal changes in brain state.

Considerable input information is also conveyed by finerscale $\mathrm{mf} V_{m}$ fluctuations (synaptic activity): slow fluctuations and fine-scale activity effectively behave as independent channels, conveying separate messages. That frequencies either side of 20 $\mathrm{Hz}$ convey separate information is consistent with LFP results in the primary visual cortex (Belitski et al., 2008). This independence could be attributable to a different biological origin for each signal. For example, each channel could reflect a different activation mode of subcortical sensory inputs, corresponding to different categories of stimulus events or features. Synchronous thalamocortical activation by strong stimulus events could trigger or modulate slow fluctuations (MacLean et al., 2005; Bruno and Sakmann, 2006; Hasenstaub et al., 2007; Reig and SanchezVives, 2007; Rigas and Castro-Alamancos, 2007; Temereanca et al., 2008; Hirata and Castro-Alamancos, 2010). Meanwhile, finer-scale signals could stem from activation of a smaller fraction of afferents, in response to different, more frequent stimulus events. Alternatively, because the finer-scale signal includes the gamma oscillation range, fine-scale information could be related to stimulus modulation of gamma rhythms (Fries et al., 2007). Concerning feature selectivity-the identity of the stimulus properties that the information is about-an important issue for future investigation is whether separate channels correspond to true multidimensional encoding, with several stimulus features being represented at the same time (e.g., whisker position and velocity), or to a situation in which one channel encodes historydependent effects (e.g., the time interval since the previous effective stimulus) or other modulatory influences (for review, see Schroeder and Lakatos, 2009; Panzeri et al., 2010).

That slow fluctuations and fine-scale synaptic activity convey comparable, essentially independent information raises an apparent inconsistency. One might expect the overall $\mathrm{mf} V_{m}$ signal, which was the sum of the slow and fine-scale signals, to convey information close to the sum of that in the two components, i.e., 
more than that carried by either slow fluctuations or fine-scale activity, yet we found no gain in information in the overall $\mathrm{mf} V_{m}$ signal compared with the $20 \mathrm{~Hz}$ low-pass filtered signal (Fig. 3D). This can be resolved by recalling that estimating information for slow and fine-scale signals required histograms with different resolution. The lower resolution used for the $\mathrm{mfV}_{m}$ and slow signals was insufficient to pick up stimulus-related structure in the fine-scale signal; conversely, the higher resolution used for estimating fine-scale information could not be used for estimating full $\mathrm{mf} V_{m}$ information, because this would have entailed an increased $N_{R}$ and poorer control over bias artifacts. Thus, the fine-scale signal, although present in the overall $\mathrm{mf} V_{m}$, needs to be separated out from the larger fluctuations to be effectively read out.

It follows that, to access different information carried by lowand high-frequency channels, a neuron has to be able to read out the separate channels. Is this operation biologically plausible? Substantial evidence suggests that the multiplexed information in different frequency bands is likely accessible to neurons through their mechanisms for synaptic integration, for example, through changes in integration time constant or spike threshold (Azouz and Gray, 1999, 2003; Azouz, 2005; Léger et al., 2005; Prescott and De Koninck, 2005; Higgs et al., 2006; Díaz-Quesada et al., 2008). Similarly, neurons may be sensitive to a specific feature (such as phase or amplitude) within a frequency channel. For example, different phases of slow oscillation can correspond to up, down, or transition states, which directly affect neuronal sensitivity (Hasenstaub et al., 2007).

\section{Information in spike timing with respect to fluctuations}

Although considerable information $(\sim 1 \mathrm{bit} / \mathrm{s})$ is carried in spike counts, spike timing relative to the stimulus significantly increases information. Furthermore, information is also increased by referencing spikes to the value at the time of firing of either the phase of slow fluctuations or the fine-scale filtered membrane potential.

The result that spike times with respect to the phase of slow membrane potential fluctuations carry information provides a cellular correlate of the spike-phase coding observed with LFPs: spike timing relative to fluctuations on different timescales potentially allows for multiplexing of information about different stimulus features (for review, see Panzeri et al., 2010). For example, in the visual thalamus, spike timing relative to the stimulus $(<30 \mathrm{~Hz})$ carries information about local changes in the visual field, whereas timing relative to retinal oscillations $(40-80 \mathrm{~Hz})$ appears to provide contextual information about global properties of the scene (Koepsell et al., 2009).

Slow membrane potential phase is shared across local cortical networks, correlates closely with LFP phase (see above), and can also correlate strongly across nearby neurons (Lampl et al., 1999; Poulet and Petersen, 2008). Thus, when a neuron spikes, its membrane potential phase is likely to be highly correlated with that of the nearby neurons to which it projects. This would allow those downstream neurons to refer the resulting EPSPs to their own phase, thereby boosting the information inherent to the spike message. Hence, the information content of spike-phase codes may be shared within local cortical networks. Whether a similar shared code might be provided by spikes labeled with higher-frequency fine-scale membrane potential fluctuations depends on whether those fluctuations are also correlated across local neurons; this requires additional exploration.

\section{Effect of up and down states}

During prolonged whisker stimulation, information transfer in up states is much richer than in down states. This is primarily attributable to the decreased firing probability in down states, because each spike within down states is comparatively information rich.

Previous comparisons of subthreshold responses within down and up states concentrated on synaptic responses evoked by the onset of a discrete stimulus (Petersen et al., 2003; Sachdev et al., 2004; Hasenstaub et al., 2007; Reig and Sanchez-Vives, 2007). Our approach differs in that, first, rather than examining individual synaptic responses, we focused on the functional outcome of synaptic activation, namely, stimulus representation by the membrane potential and by spikes; second, we investigated ongoing responses during prolonged periods of stimulation, intended to parallel extended periods of irregular, complex whisker motion. Overall evidence from previous work is consistent with our findings in that, although individual synaptic responses tend to be reduced during up states, the overall spiking responsiveness to a stimulus increases (Rigas and Castro-Alamancos, 2009). During up states, neurons are more responsive and less selective, generating the overwhelming majority of spikes; changes between down and up states modulate feature tuning, because the precise nature of the stimulus features encoded in spiking is state dependent (Hasenstaub et al., 2007). Our results appear to differ from in vitro evidence that cortical activity is insensitive to thalamic input during up states (Watson et al., 2008); this may be because of differences between slice and in vivo conditions. Finally, we note that the present form of up-down oscillations is not found in awake behaving animals; instead, up states could be "fragments of wakefulness," corresponding to activated sensory awareness (Destexhe et al., 2007).

\section{Measuring intracellular information in vivo}

Applying information methods to membrane potential signals bypasses the explicit extraction of synaptic responses (Pospischil et al., 2009) and focuses directly on the functional outcome of synaptic activation. Here we have shown that, with appropriate bias reduction, direct information estimates are feasible for cortical in vivo membrane potential data. We expect this approach to be applicable to other experimental designs: information provides a metric for comparing sensory coding across different test conditions (Sohal et al., 2009) or developmental stages.

One interesting topic for future work is whether there are differences in sensory information across neurons in different layers and positions within cortical microcircuits. Our present main conclusions (independence of information in different frequency ranges; information gain when considering spike timing relative to the stimulus and to fluctuations) held regardless of recording depth (data not shown).

\section{References}

Ahissar E, Vaadia E (1990) Oscillatory activity of single units in a somatosensory cortex of an awake monkey and their possible role in texture analysis. Proc Natl Acad Sci U S A 87:8935-8939.

Amzica F, Steriade M (1995) Short- and long-range neuronal synchronization of the slow $(<1 \mathrm{~Hz})$ cortical oscillation. J Neurophysiol 73:20-38.

Anderson J, Lampl I, Reichova I, Carandini M, Ferster D (2000) Stimulus dependence of two-state fluctuations of membrane potential in cat visual cortex. Nat Neurosci 3:617-621.

Arabzadeh E, Zorzin E, Diamond ME (2005) Neuronal encoding of texture in the whisker sensory pathway. PLoS Biol 3:e17.

Arieli A, Shoham D, Hildesheim R, Grinvald A (1995) Coherent spatiotemporal patterns of ongoing activity revealed by real-time optical imaging 
coupled with single-unit recording in the cat visual cortex. J Neurophysiol 73:2072-2093.

Arieli A, Sterkin A, Grinvald A, Aertsen A (1996) Dynamics of ongoing activity: explanation of the large variability in evoked cortical responses. Science 273:1868-1871.

Azouz R (2005) Dynamic spatiotemporal synaptic integration in cortical neurons: neuronal gain, revisited. J Neurophysiol 94:2785-2796.

Azouz R, Gray CM (1999) Cellular mechanisms contributing to response variability of cortical neurons in vivo. J Neurosci 19:2209-2223.

Azouz R, Gray CM (2003) Adaptive coincidence detection and dynamic gain control in visual cortical neurons in vivo. Neuron 37:513-523.

Belitski A, Gretton A, Magri C, Murayama Y, Montemurro MA, Logothetis NK, Panzeri S (2008) Low-frequency local field potentials and spikes in primary visual cortex convey independent visual information. J Neurosci 28:5696-5709.

Borst A, Theunissen FE (1999) Information theory and neural coding. Nat Neurosci 2:947-957.

Bruno RM, Sakmann B (2006) Cortex is driven by weak but synchronously active thalamocortical synapses. Science 312:1622-1627.

Buonomano DV, Maass W (2009) State-dependent computations: spatiotemporal processing in cortical networks. Nat Rev Neurosci 10:113-125.

Buracas GT, Zador AM, DeWeese MR, Albright TD (1998) Efficient discrimination of temporal patterns by motion-sensitive neurons in primate visual cortex. Neuron 20:959-969.

Buzsáki G (2006) Rhythms of the brain. New York: Oxford UP.

Castro-Alamancos MA (2004) Absence of rapid sensory adaptation in neocortex during information processing states. Neuron 41:455-464.

Cowan RL, Wilson CJ (1994) Spontaneous firing patterns and axonal projections of single corticostriatal neurons in the rat medial agranular cortex. J Neurophysiol 71:17-32.

de Ruyter van Steveninck RR, Lewen GD, Strong SP, Koberle R, Bialek W (1997) Reproducibility and variability in neural spike trains. Science 275:1805-1808.

Destexhe A, Contreras D (2006) Neuronal computations with stochastic network states. Science 314:85-90.

Destexhe A, Hughes SW, Rudolph M, Crunelli V (2007) Are corticothalamic "up" states fragments of wakefulness? Trends Neurosci 30:334-342.

DeWeese MR (2007) Whole-cell recording in vivo. Curr Protoc Neurosci 6:6.22.

Deweese MR, Zador AM (2004) Shared and private variability in the auditory cortex. J Neurophysiol 92:1840-1855.

Díaz-Quesada M, Maravall M (2008) Intrinsic mechanisms for adaptive gain rescaling in barrel cortex. J Neurosci 28:696-710.

Erchova IA, Lebedev MA, Diamond ME (2002) Somatosensory cortical neuronal population activity across states of anaesthesia. Eur J Neurosci $15: 744-752$.

Fiser J, Chiu C, Weliky M (2004) Small modulation of ongoing cortical dynamics by sensory input during natural vision. Nature 431:573-578.

Friedrich RW, Habermann CJ, Laurent G (2004) Multiplexing using synchrony in the zebrafish olfactory bulb. Nat Neurosci 7:862-871.

Fries P, Nikolić D, Singer W (2007) The gamma cycle. Trends Neurosci 30:309-316.

Gao L, Meng X, Ye C, Zhang H, Liu C, Dan Y, Poo MM, He J, Zhang X (2009) Entrainment of slow oscillations of auditory thalamic neurons by repetitive sound stimuli. J Neurosci 29:6013-6021.

Hasenstaub A, Sachdev RN, McCormick DA (2007) State changes rapidly modulate cortical neuronal responsiveness. J Neurosci 27:9607-9622.

Haslinger R, Ulbert I, Moore CI, Brown EN, Devor A (2006) Analysis of LFP phase predicts sensory response of barrel cortex. J Neurophysiol 96:1658-1663.

Higgs MH, Slee SJ, Spain WJ (2006) Diversity of gain modulation by noise in neocortical neurons: regulation by the slow afterhyperpolarization conductance. J Neurosci 26:8787-8799.

Hirata A, Castro-Alamancos MA (2010) Neocortex network activation and deactivation states controlled by the thalamus. J Neurophysiol 103:1147-1157.

Hopfield JJ (1995) Pattern recognition computation using action potential timing for stimulus representation. Nature 376:33-36.

Huxter J, Burgess N, O'Keefe J (2003) Independent rate and temporal coding in hippocampal pyramidal cells. Nature 425:828-832.

Huxter JR, Senior TJ, Allen K, Csicsvari J (2008) Theta phase-specific codes for two-dimensional position, trajectory and heading in the hippocampus. Nat Neurosci 11:587-594.

Jadhav SP, Wolfe J, Feldman DE (2009) Sparse temporal coding of elementary tactile features during active whisker sensation. Nat Neurosci 12:792-800.

Jones LM, Fontanini A, Sadacca BF, Miller P, Katz DB (2007) Natural stimuli evoke dynamic sequences of states in sensory cortical ensembles. Proc Natl Acad Sci U S A 104:18772-18777.

Juusola M, de Polavieja GG (2003) The rate of information transfer of naturalistic stimulation by graded potentials. J Gen Physiol 122:191-206.

Kayser C, Montemurro MA, Logothetis NK, Panzeri S (2009) Spike-phase coding boosts and stabilizes information carried by spatial and temporal spike patterns. Neuron 61:597-608.

Kenet T, Bibitchkov D, Tsodyks M, Grinvald A, Arieli A (2003) Spontaneously emerging cortical representations of visual attributes. Nature 425:954-956.

Koepsell K, Wang X, Vaingankar V, Wei Y, Wang Q, Rathbun DL, Usrey WM, Hirsch JA, Sommer FT (2009) Retinal oscillations carry visual information to cortex. Front Syst Neurosci 3:4.

Koepsell K, Wang X, Hirsch JA, Sommer FT (2010) Exploring the function of neural oscillations in early sensory systems. Front Neurosci 4:53-61.

Lampl I, Reichova I, Ferster D (1999) Synchronous membrane potential fluctuations in neurons of the cat visual cortex. Neuron 22:361-374.

Léger JF, Stern EA, Aertsen A, Heck D (2005) Synaptic integration in rat frontal cortex shaped by network activity. J Neurophysiol 93:281-293.

Lisman J (2005) The theta/gamma discrete phase code occuring during the hippocampal phase precession may be a more general brain coding scheme. Hippocampus 15:913-922.

Loewenstein Y, Mahon S, Chadderton P, Kitamura K, Sompolinsky H, Yarom Y, Häusser M (2005) Bistability of cerebellar Purkinje cells modulated by sensory stimulation. Nat Neurosci 8:202-211.

Lottem E, Azouz R (2008) Dynamic translation of surface coarseness into whisker vibrations. J Neurophysiol 100:2852-2865.

Lottem E, Azouz R (2009) Mechanisms of tactile information transmission through whisker vibrations. J Neurosci 29:11686-11697.

Luczak A, Barthó P, Harris KD (2009) Spontaneous events outline the realm of possible sensory responses in neocortical populations. Neuron 62: $413-425$.

MacLean JN, Watson BO, Aaron GB, Yuste R (2005) Internal dynamics determine the cortical response to thalamic stimulation. Neuron 48: 811-823.

Maravall M, Petersen RS, Fairhall AL, Arabzadeh E, Diamond ME (2007) Shifts in coding properties and maintenance of information transmission during adaptation in barrel cortex. PLoS Biol 5:e19.

Margrie TW, Brecht M, Sakmann B (2002) In vivo, low-resistance, wholecell recordings from neurons in the anaesthetized and awake mammalian brain. Pflugers Arch 444:491-498.

Metherate R, Ashe JH (1993) Ionic flux contributions to neocortical slow waves and nucleus basalis-mediated activation: whole-cell recordings in vivo. J Neurosci 13:5312-5323.

Montemurro MA, Senatore R, Panzeri S (2007) Tight data-robust bounds to mutual information combining shuffling and model selection techniques. Neural Comput 19:2913-2957.

Montemurro MA, Rasch MJ, Murayama Y, Logothetis NK, Panzeri S (2008) Phase-of-firing coding of natural visual stimuli in primary visual cortex. Curr Biol 18:375-380.

Nadasdy Z (2009) Information encoding and reconstruction from the phase of action potentials. Front Syst Neurosci 3:6.

O’Keefe J, Recce ML (1993) Phase relationship between hippocampal place units and the EEG theta rhythm. Hippocampus 3:317-330.

Panzeri S, Treves A (1996) Analytical estimates of limited sampling biases in different information measures. Network 7:87-107.

Panzeri S, Senatore R, Montemurro MA, Petersen RS (2007) Correcting for the sampling bias problem in spike train information measures. J Neurophysiol 98:1064-1072.

Panzeri S, Brunel N, Logothetis NK, Kayser C (2010) Sensory neural codes using multiplexed temporal scales. Trends Neurosci 33:111-120.

Petersen CC, Hahn TT, Mehta M, Grinvald A, Sakmann B (2003) Interaction of sensory responses with spontaneous depolarization in layer $2 / 3$ barrel cortex. Proc Natl Acad Sci U S A 100:13638-13643.

Petersen RS, Panzeri S, Maravall M (2009) Neural coding and contextual influences in the whisker system. Biol Cybern 100:427-446. 
Pospischil M, Piwkowska Z, Bal T, Destexhe A (2009) Extracting synaptic conductances from single membrane potential traces. Neuroscience 158:545-552.

Poulet JF, Petersen CC (2008) Internal brain state regulates membrane potential synchrony in barrel cortex of behaving mice. Nature 454:881-885.

Prescott SA, De Koninck Y (2005) Integration time in a subset of spinal lamina I neurons is lengthened by sodium and calcium currents acting synergistically to prolong subthreshold depolarization. J Neurosci 25:4743-4754.

Quian Quiroga R, Panzeri S (2009) Extracting information from neuronal populations: information theory and decoding approaches. Nat Rev Neurosci 10:173-185.

Reig R, Sanchez-Vives MV (2007) Synaptic transmission and plasticity in an active cortical network. PLoS One 2:e670.

Rigas P, Castro-Alamancos MA (2007) Thalamocortical up states: differential effects of intrinsic and extrinsic cortical inputs on persistent activity. J Neurosci 27:4261-4272.

Rigas P, Castro-Alamancos MA (2009) Impact of persistent cortical activity (up states) on intracortical and thalamocortical synaptic inputs. J Neurophysiol 102:119-131.

Ringach DL (2009) Spontaneous and driven cortical activity: implications for computation. Curr Opin Neurobiol 19:439-444.

Ritt JT, Andermann ML, Moore CI (2008) Embodied information processing: vibrissa mechanics and texture features shape micromotions in actively sensing rats. Neuron 57:599-613.

Sachdev RN, Ebner FF, Wilson CJ (2004) Effect of subthreshold up and down states on the whisker-evoked response in somatosensory cortex. J Neurophysiol 92:3511-3521.

Saleem AB, Chadderton P, Apergis-Schoute J, Harris KD, Schultz SR (2010)
Methods for predicting cortical UP and DOWN states from the phase of deep layer local field potentials. J Comput Neurosci. Advance online publication. Retrieved March 12, 2010. doi:10.1007/s10827-010-0228-5.

Schroeder CE, Lakatos P (2009) Low-frequency neuronal oscillations as instruments of sensory selection. Trends Neurosci 32:9-18.

Shannon C (1948) A mathematical theory of communication. Bell Sys Tech J 27:379-423.

Sohal VS, Zhang F, Yizhar O, Deisseroth K (2009) Parvalbumin neurons and gamma rhythms enhance cortical circuit performance. Nature 459:698-702.

Steriade M, Nuñez A, Amzica F (1993) A novel slow $(<1 \mathrm{~Hz})$ oscillation of neocortical neurons in vivo: depolarizing and hyperpolarizing components. J Neurosci 13:3252-3265.

Strong SP, Koberle R, de Ruyter van Steveninck R, Bialek W (1998) Entropy and information in neural spike trains. Phys Rev Lett 80:197-200.

Temereanca S, Brown EN, Simons DJ (2008) Rapid changes in thalamic firing synchrony during repetitive whisker stimulation. J Neurosci 28:11153-11164.

Tsodyks M, Kenet T, Grinvald A, Arieli A (1999) Linking spontaneous activity of single cortical neurons and the underlying functional architecture. Science 286:1943-1946.

Watson BO, MacLean JN, Yuste R (2008) UP states protect ongoing cortical activity from thalamic inputs. PLoS One 3:e3971.

Wolfe J, Hill DN, Pahlavan S, Drew PJ, Kleinfeld D, Feldman DE (2008) Texture coding in the rat whisker system: slip-stick versus differential resonance. PLoS Biol 6:e215.

Zohary E, Shadlen MN, Newsome WT (1994) Correlated neuronal discharge rate and its implications for psychophysical performance. Nature 370:140-143. 